\title{
Aggregation of nanoparticles on one and two-component bilayer membranes
}

\author{
David L. Cheung \\ Department of Pure and Applied Chemistry, \\ University of Strathclyde, Glasgow, G1 $1 X L$, UK* \\ Abstract
}

Using dissipative particle dynamics simulations the aggregation of nanoparticles on single and two-component bilayers is investigated. For a uniform bilayer the aggregation of nanoparticles depends strongly on the location of the particles in the bilayer; particles residing on the bilayer exterior cluster strongly under the influence of bilayer-mediated interactions, whereas the interaction between the particles in the bilayer interior is significantly weaker leading to more loosely bound, dynamic aggregates. The aggregation of nanoparticles on two-component bilayers composed of immiscible components changes due to competition between nanoparticle clustering and their adsorption on the boundary between the bilayer components. This reduces the size of the nanoparticle clusters formed on the bilayer exterior, with the clusters adhering onto the boundary between the bilayer components. Due to their weaker attraction nanoparticles in the interior of a mixed bilayer no longer aggregate and instead form strings along the boundary between the two bilayer components. Nanoparticles with an affinity to one bilayer component nucleate small domains of their favoured component around themselves. For asymmetric mixtures this leads to a notable change in the aggregation behaviour of the nanoparticles. 


\section{INTRODUCTION}

Polymer vesicles ${ }^{1}$, fluid filled sacs formed by polymer bilayers, may be considered as nanometre scale containers and have attracted much attention over the past decade for a range potential and current applications. In contrast to other polymer nanostructures, such as micelles, they can encapsulate both hydrophilic and hydrophobic molecules (in the fluid filled interior and core of the bilayer respectively) giving them more flexibility in delivery applications. Due to the larger size of the constituent molecules polymer vesicles possess higher mechanical stability than those formed by small lipid molecules and the properties of polymer vesicles may be further controlled through changes to polymer length.

As polymer vesicles consist of a bilayer enclosing a sac of fluid they are often thought of as being minimal models of biological cells. In comparison to synthetic bilayers, biological membranes possess a large degree of extra complexity. Rather than being a simple bilayer, the cell membrane is a complex mixture of different lipid types, as well as containing a variety of non-lipid molecules, such as cholesterol. The cell membrane is further complicated by the presence of membrane proteins, both attached to and embedded within it. There are also a number of additional structures that nature builds on top of the cell membrane. One example are the shells of inorganic nanoparticles that are built on the outside of many microogranisms such as calcium carbonate shells on the outside of coccoliths ${ }^{2}$ and silica shells on diatoms. Many bacterial produce similar structures though the formation of crystalline protein layers (so-called S-layers $)^{3}$ on the exterior of their outer membranes.

In recent years there have been a number of studies aimed at incorporating some degree of this biological complexity into synthetic systems ${ }^{4}$. One notable example was the work of Christain et al who studied polymer vesicles and micelles formed from mixtures of poly(acrylic-acid)-poly(butadiene) and poly(ethyleneoxide)-poly(butadiene) copolymers ${ }^{5}$. Addition of calcium ions leads to strong lateral phase separation in the vesicles, causing the appearance of patchy and Janus vesicles, depending on the vesicle composition and solution conditions. Using coarse-grain molecular dynamics simulations Pantano et al rationalised this due to local structural rearrangements caused by the polyvalent calcium ions ${ }^{6}$. The patchy structure, with feature sizes on the order of microns, is reminiscent of domains that have been observed in lipid vesicles create in vitro. These domains are significantly larger than those found in cell membranes. The disparity in size may be attributable to mem- 
brane proteins nucleating or stabilising these small domains, non-equilibrium recycling ${ }^{7}$ or coupling between membrane curvature and domain formation ${ }^{8}$.

The adhesion of particles onto polymer vesicles has also attracted attention ${ }^{9}$. Chen et al showed it is possible to create armoured vesicles by adhering negitively charged colloidal particles onto positively charged polymer vesicles ${ }^{10}$. Simple Monte Carlo simulations were used to study the packing of binary mixtures of particles on the vesicles, pointing out the key role played by electrostatic repulsion between particles on their packing. For smaller particles adhesion onto vesicles can also lead to changes in the bilayer structure. Binder et al showed that by changing nanoparticle hydrophobicity it is possible to guide the location of these in the vesicle ${ }^{11}$. Through adding anionic nanoparticles onto phospholipid vesicles Wang et al showed that it was possible to induce reconstruction of the vesicle and formation of patchy domains ${ }^{12}$, similar to the work of Christian et $a l^{5}$. While these studies have examined single component vesicles recently it has been generalised to mixed vesicles which exhibit macroscopic phase separation. When membrane binding proteins are added to these mixed vesicles their structure changes, reflecting a decrease in the line tension between the different domains ${ }^{13}$. This was attributed to the proteins adhering to the interface between the different lipid domains and reducing the interfacial free energy, in a manner reminiscent of the adhesion of colloids or nanoparticles onto liquid-liquid interfaces in the formation of Pickering emulsions ${ }^{14}$.

A number of molecular simulation studies have been performed on nanoparticle-bilayer systems. Due to the interest in the use of nanoparticles in biomedical applications ${ }^{15}$, such as drug delivery or medical imaging, and on the potential toxic effects of nanomaterials, many of these have focused on how nanoparticles penetrate bilayers. Much of this work has focused on the translocation of individual nanoparticles ${ }^{16-18}$ or changes to bilayer structure induced by nanoparticles ${ }^{19}$. The collective behaviour of nanoparticles has also been studied. In particular aggregation of nanoparticles onto fluid bilayers has been shown to lead to stabilisation of membrane pores $^{20}$ and endocytosis ${ }^{21}$. Recently attention has been paid to the self-assembly of nanoparticles on bilayers, focusing on particles larger than the bilayer width ${ }^{22}$. In these cases aggregation of particles into both compact (hexagonal) arrays and extended linear aggregates has been seen in simulation, depending on the bilayer rigidity and particle size $\mathrm{s}^{21,23}$. Elongated linear structures are also predicted for anisotropic particles $^{24}$, driven by the anisotropy in the particle-particle interactions. Matthews and 
Likos have investigated effect of membrane fluctuations on self-assembly of virus capsids ${ }^{25}$ and clathrin ${ }^{26}$. Most closely related to this paper Reynwar and Desereno ${ }^{27}$ have studied the aggregation of model proteins on a symmetric two-component bilayer close to demixing and found composition-driven formation of protein clusters.

In this paper molecular simulation is used to study the aggregation of small $(\sim n m)$ nanoparticles on model bilayer membranes. The aggregation of nanoparticles on uniform polymer bilayers will be discussed, focusing on how the location within the bilayer affects the clustering of the nanoparticles. Next the aggregation of nanoparticles on phase separated bilayers will be examined, in particular showing that these tend to aggregate on the boundary between the two components. Finally the behaviour of nanoparticles on bilayers composed of two miscible components is investigated.

\section{SIMULATION DETAILS}

\section{A. Methodology}

The system was simulated using dissipative particle dynamics simulations (DPD) ${ }^{28}$. The interaction between two beads is given by

$$
\boldsymbol{F}_{i j}=\boldsymbol{F}_{i j}^{c}+\boldsymbol{F}_{i j}^{D}+\boldsymbol{F}_{i j}^{R}
$$

where the right hand terms are the conservative, dissipative, and random forces respectively.

The conservative force is given by the usual soft, repulsive form ${ }^{29}$

$$
\boldsymbol{F}_{i j}=A_{i j}\left[1-\left(\frac{r}{r_{c}}\right)\right] \hat{\boldsymbol{r}}_{i j}=A_{i j} w(r) \hat{\boldsymbol{r}}_{i j}
$$

where $A_{i j}$ is the repulsion strength between two beads (see below), $r=\left|\boldsymbol{r}_{i j}\right|$, and $r_{c}$ is the bead size. To enforce the polymer topology bonds between bonded monomers are modelled using harmonic springs

$$
F_{\text {spring }}=-k \boldsymbol{r}_{i j}
$$

where $k=2 k_{\mathrm{B}} \operatorname{Tr}_{c}^{-2}$ is the force constant. Note no bond angle bending potentials are applied. 
$\boldsymbol{F}_{i j}^{D}$ and $\boldsymbol{F}_{i j}^{R}$ are given by

$$
\begin{aligned}
\boldsymbol{F}_{i j}^{D} & =-\gamma w^{2}(r)\left(\hat{\boldsymbol{r}}_{i j} . \boldsymbol{v}_{i j}\right) \hat{\boldsymbol{r}}_{i j} \\
\boldsymbol{F}_{i j}^{R} & =\frac{\sigma w(r) R}{\sqrt{\delta t}} \hat{\boldsymbol{r}}_{i j}
\end{aligned}
$$

where $\gamma$ is the damping coefficient $\left(\gamma=4.5 k_{\mathrm{B}} T \tau r_{c}^{-2}\right), \sigma=\sqrt{2 k_{\mathrm{B}} T \gamma}$ is the random force strength, $R$ is a Gaussian random number (with zero mean and variance of one), and $\delta t$ is the timestep.

\section{B. Model}

The simulated system contained 144000 beads in total in a cuboidal box measuring $40 r_{c} \times 40 r_{c} \times 30 r_{c}$ (corresponding to a number density $\rho=3 r_{c}^{-3}$ ). It contained a single bilayer consisting of $1920 \mathrm{H}_{8} \mathrm{~T}_{12}$ polymer chains (960 in each leaflet, which corresponds to the zero surface tension state for a single component bilayer) and up to 80 nanoparticles, corresponding to between 24 and 192 polymer chains per nanoparticle, comparable to previous work ${ }^{20}$. For particles that reside on the bilayer exterior these were split equally between the two leaflets. Each nanoparticle was a rigid FCC array of interaction sites of radius $1.5 r_{c}$ (containing 44 interaction sites in total). The spacing between the interaction sites $\left(0.55 r_{c}\right)$ was sufficient to ensure that no solvent beads entered the nanoparticle interior.

The interaction parameters were derived from those of Groot and Rabone ${ }^{30}$. In all cases the repulsion strength between identical particles was set to $A_{i i}=78 k_{\mathrm{B}} T r_{c}^{-1}$, while for interactions between the solvent and head, tail, and nanoparticle the repulsion parameter was set to $A_{H S}=79.3 k_{\mathrm{B}} \operatorname{Tr}_{c}^{-1}, A_{T S}=104 k_{\mathrm{B}} \operatorname{Tr}_{c}^{-1}$ and $A_{N S}=90 k_{\mathrm{B}} \operatorname{Tr}_{c}^{-1}$, while for the head-tail interaction $A_{H T}=86.7 k_{\mathrm{B}} \operatorname{Tr}_{c}^{-1}\left(r_{c}\right.$ is the same for all interactions, which following the mapping of Groot and Rabone $r_{c} \sim 6.46 \AA$ ). To model two-component bilayers the repulsion strength between either the head or tail beads in unlike polymers is increased (with the other repulsion parameters unchanged). For unlike head groups $A_{H H^{\prime}}$ values of $90 k_{\mathrm{B}} \operatorname{Tr}_{c}^{-1}$ or $80 k_{\mathrm{B}} \operatorname{Tr}_{c}^{-1}$ were used, while for unlike tail groups $A_{T T^{\prime}}$ values of $85 k_{\mathrm{B}} \operatorname{Tr}_{c}^{-1}$ or $79 k_{\mathrm{B}} \operatorname{Tr}_{c}^{-1}$ were used. The values of the repulsion parameters for interactions between unlike polymers were chosen to be sufficiently far from the critical values for phase separation, regardless of the bilayer composition. $A_{T T^{\prime}}=90 k_{\mathrm{B}} \operatorname{Tr}_{c}^{-1}$ the bilayer with unlike tails is unstable in the absence of nanoparticles, so a lower value (compared to unlike heads) was used in this 
case. It should be noted that while these values were not chosen to match a specific experimental system a number of schemes for determining DPD interaction parameters from experimental data ${ }^{31,32}$ which generally give values for bead-bead repulsion similar to these. Two different bilayer compositions were investigated, $x_{b}=0.50$ (symmetric) and $x_{b}=0.25$ (asymmetric). Simulations of mixed bilayers in the absence of nanoparticles indicate that phase separation between the two components is seen for $A_{H H^{\prime}}=84 k_{\mathrm{B}} \operatorname{Tr}_{c}^{-1}\left(x_{b}=0.50\right)$ and $A_{H H^{\prime}}=85 k_{\mathrm{B}} \operatorname{Tr}_{c}^{-1}\left(x_{b}=0.25\right)$ for unlike heads and $A_{T T^{\prime}}=82 k_{\mathrm{B}} \operatorname{Tr}_{c}^{-1}\left(x_{b}=0.50\right)$ and $A_{T T^{\prime}}=84 k_{\mathrm{B}} \operatorname{Tr}_{c}^{-1}\left(x_{b}=0.25\right)$ for unlike tails. Throughout this paper the two components will be referred to as A and B (where in the asymmetric mixture B is the minority component) respectively.

Four different types of nanoparticles were considered depending on their preferred location in the bilayer (either in the bilayer exterior or interior) and affinity for the bilayer components (either neutral or with a preference for the minority B component). To ensure the location of the nanoparticles in their preferred location the repulsion parameter between the nanoparticle beads and their preferred component is set to $78 k_{\mathrm{B}} T r_{c}^{-1}$ (i.e. the nanoparticle is treated as chemically identical to its preferred component) while the repulsion parameters for the other interactions are set to $90 k_{\mathrm{B}} \operatorname{Tr}_{c}^{-1}$ which was large enough to restrict them to the bilayer interior or exterior as appropriate. The interaction parameters for each of these cases are given in Table I.

\begin{tabular}{ll}
\hline Particle type & Interaction parameters \\
\hline Neutral, exterior (NE) & $A_{N H}=A_{N H^{\prime}}=78 k_{\mathrm{B}} T r_{c}^{-1}, A_{N T}=A_{N T^{\prime}}=90 k_{\mathrm{B}} T r_{c}^{-1}$ \\
Neutral, interior (NI) & $A_{N H}=A_{N H^{\prime}}=90 k_{\mathrm{B}} T r_{c}^{-1}, A_{N T}=A_{N T^{\prime}}=78 k_{\mathrm{B}} T r_{c}^{-1}$ \\
B-philic, exterior (BE) & $A_{N H^{\prime}}=78 k_{\mathrm{B}} T r_{c}^{-1}, A_{N H}=A_{N T}=A_{N T^{\prime}}=90 k_{\mathrm{B}} T r_{c}^{-1}$ \\
B-philic, interior (BI) & $A_{N T^{\prime}}=78 k_{\mathrm{B}} \operatorname{Tr}_{c}^{-1}, A_{N H}=A_{N H^{\prime}}=A_{N T}=90 k_{\mathrm{B}} T r_{c}^{-1}$ \\
\hline
\end{tabular}

TABLE I. Interaction parameters for nanoparticle-polymer interactions.

For all systems the initial state was a flat bilayer with polymers randomly placed in the $x y$ plane in a fully extended configuration. Nanoparticles were randomly inserted in the bilayer at the boundary between the head and tail blocks. For mixed bilayers the two components were placed randomly (corresponding to an initially well mixed state). A short energy minimisation and DPD run (with uniform interaction parameters) was performed before the 
repulsion between unlike polymers was applied. For each system three simulations starting from different initial configurations were performed, with final results obtained by averaging over these. Each simulation was run for $10^{6}-2 \times 10^{6}$ timesteps, where a time step $\delta t=0.02 t^{*}$, was used. Following Groot and Rabone $t^{*}=88$ ps. All simulations were performed using the LAMMPs molecular dynamics package ${ }^{33}$. It should be noted that in these simulations the cross-sectional area of the bilayer was held constant. While simulations of bilayers at constant surface tension are commonly used, only minor distortions in the shape of the bilayer were observed (Fig. 1) suggesting that the results are unlikely to be qualitatively changed by this choice.

\section{NANOPARTICLE AGGREGATION ON UNIFORM BILAYERS}

While nanoparticles both on the exterior of the bilayer and in the bilayer interior have a tendency to aggregate, the degree of this is strongly dependent on the location of the nanoparticles in the bilayer (Fig. 1). For particles on the bilayer exterior cluster formation is observed for even small numbers of particles, indicating a strong attraction between the particles. By contrast for small $N_{\text {par }}$ particles in the bilayer interior show little tendency to aggregate, reflecting a weaker interaction between the particles in the interior. Side on views of the bilayers show that the addition of particles causes little distortion to the bilayer; in particular the bilayer appears approximately flat for the different systems studied.

The aggregation of particles on the bilayer may be examined more quantitatively through radial distribution functions and histograms of nanoparticle cluster sizes. Due to the quasitwo-dimensional nature of the bilayer throughout this paper we only consider the in-plane RDF (as a function of the separation between nanoparticles in the plane of the bilayer). The tendency of nanoparticles to cluster strongly may be seen from the large peak in the in-plane RDF (Fig. 2(a)), which is largest for small particle numbers. Increasing $N_{\text {par }}$ reduces the height of this peak. There is also some additional structure at larger $r$ for larger particle number, which appears somewhat diffuse. This indicates that there is a lack of long-range order, suggesting that these larger clusters have a disordered structure.

For particles in the bilayer interior the behaviour is quite different (Fig. 2(b)). For smaller particle numbers little structure is seen in the RDF; this has a single peak at about 1 particle diameter and tends rapidly to constant after this. This indicates that at these lower density 

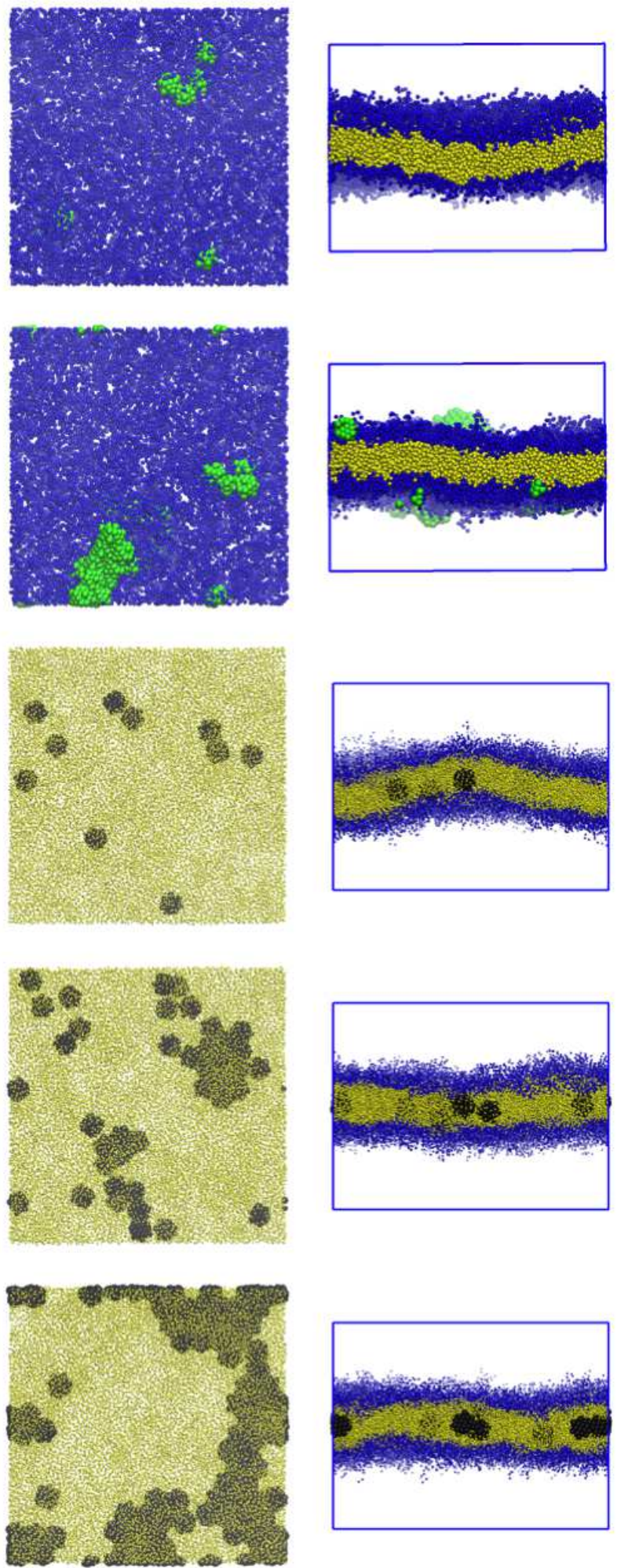

FIG. 1. Simulation snapshots showing representative configurations for (top to bottom) NE nanoparticles with $N_{p a r}=10$, NE nanoparticles with $N_{p a r}=40$, NI nanoparticles with $N_{p a r}=10$, NI nanoparticles with $N_{\text {par }}=40$, and NI nanoparticles with $N_{\text {par }}=80$. Left hand column shows top down view, right hand column side view. \$n all cases blue, yellow, green, and black spheres denote head, tail, exterior, and interior nanoparticles respectively. 

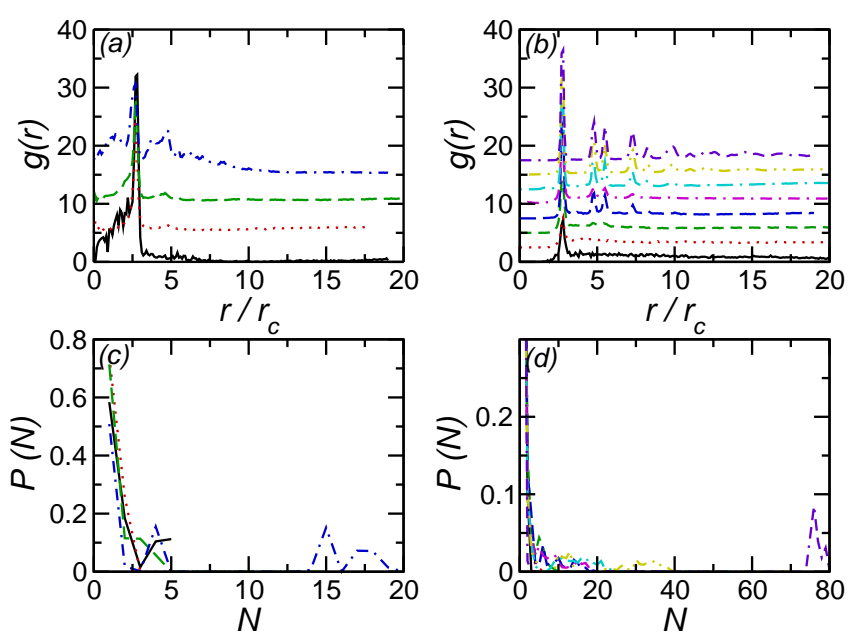

FIG. 2. (a) RDF for NE nanoparticles on uniform bilayer. Solid line (black) denotes $N_{\text {par }}=10$, dotted line (red) denotes $N_{\text {par }}=20$, dashed line (green) denotes $N_{\text {par }}=30$, and dot-dashed line denotes $N_{\text {par }}=40$. For clarity successive curves have been shifted by 5 along the $y$-axis. (b) RDF for NI nano particles on uniform bilayer. Solid line (black) denotes $N_{\text {par }}=10$, dotted line (red) denotes $N_{\text {par }}=20$, dashed line (green) denotes $N_{\text {par }}=30$, long-dashed line (blue) denotes $N_{\text {par }}=40$, dot-dashed line (magenta) denotes $N_{\text {par }}=50$, dot-long-dashed line (cyan) denotes $N_{\text {par }}=60$, double-dot-dashed line (yellow) denotes $N_{\text {par }}=70$, and dot-double-dashed line (violet) denotes $N_{\text {par }}=80$. For clarity successive curves have been shifted by 2.5 along the $y$-axis. (c) Nanoparticle cluster size histograms for NE nanoparticles on uniform bilayers. Symbols as in (a). (d) Nanoparticle cluster size histograms for NI nano particles on uniform bilayers. Symbols as in (b).

the particles behave as a $2 \mathrm{D}$ liquid. Above $N_{\text {par }}=40$ a number of well-defined peaks appear at larger separations in the RDF. The spacing between these peaks suggests local hexagonal ordering of the nanoparticles, which may be seen from the simulation snapshots (Fig. 1).

As well as the RDFs the aggregation of nanoparticles may also be investigated through the distribution of nanoparticle cluster sizes. The sizes of these clusters also depend on the number of particles as shown by the cluster size histograms (Fig. 2(b)). For all particle numbers there is a significant probability of finding isolated nanoparticles $(P(1) \sim 0.5-0.75)$. Only for $N_{\text {par }}=10$ and 40 is there an appreciable population of larger aggregates. It is noticeable that the distribution of cluster sizes tends to be quite sharp; this suggests that the particles are strongly bound into long-lived (relative to the simulation time) clusters. 
Reflecting the weaker interactions between particles the cluster size distributions for particles in the bilayer interior are broader than those for particles on the exterior (Fig. 2(d)). For small $N_{\text {par }}$ the cluster size distribution is peaked at $N=1$ with a rapid decay. As $N_{\text {par }}$ increases peaks in the distribution at larger $N$, indicating the existence of finite sized aggregates. For the largest studied $N_{\text {par }}$ the cluster size distribution suggests the formation of a single system spanning cluster (containing $\sim 75-80$ particles) are found, with a small number of isolated particles. This transition to an infinitely-large cluster above a critical density is consistent with aggregation in two-dimensional systems ${ }^{34}$.

The difference in the interaction between nanoparticles on the bilayer exterior and interior may be due to differences in the bilayer bending modulus $\kappa^{35,36}$. From simulation $\kappa$ may be extracted from the fluctuation spectrum of bilayer undulations ${ }^{37,38}$ (Table II). $\kappa$ is generally similar for bilayers with both interior and exterior nanoparticles and it tends to decrease with increasing $N_{\text {par }}$. For low particle numbers, where the analytic expressions for the bilayerinduced interactions generally valid, $\kappa$ is larger for the bilayer with exterior nanoparticles compared to the interior case, which is consistent with the stronger interaction between the nanoparticles seen in that case. The bilayer surface tension, calculated from the difference between the normal and transverse pressures are also presented in Table II. In all cases this is small suggesting that the bilayers are close to the zero surface tension state.

The difference in interactions between particles on the bilayer exterior and interior also affects how the numbers and sizes of clusters vary with time. When the particles are on the exterior both the number of clusters and largest cluster size show little variation with time, which is particularly noticeable for $N_{\text {par }}=10$ when maximum cluster size is $4-5$. This corresponds to the particles in one leaflet forming a single long-lived cluster, due to the strong interparticle interactions. For interior nanoparticles both $N_{\text {cluster }}$ and the maximum cluster size show more variation with time, reflecting that the nanoparticles are less strongly bound allowing these to dynamically join and leave clusters. The formation of a single nanoparticle cluster for the $N_{\text {par }}=80$ system can be clearly seen in the sudden jump in the maximum cluster size to $\sim 75-80$ approximately half way through the simulation. 


\begin{tabular}{ccccc}
\hline \multirow{2}{*}{$N_{\text {par }}$} & \multicolumn{2}{c}{ Exterior } & \multicolumn{2}{c}{ Interior } \\
& $\kappa / k_{\mathrm{B}} T$ & $\sigma / k_{\mathrm{B}} \operatorname{Tr}_{c}^{-2}$ & $\kappa / k_{\mathrm{B}} T$ & $\sigma / k_{\mathrm{B}} \operatorname{Tr}_{c}^{-2}$ \\
\hline 0 & $10.0(1.9)$ & $-0.12(6)$ & $10.0(1.9)$ & $-0.12(6)$ \\
10 & $10.3(2.4)$ & $-0.18(4)$ & $8.8(2.0)$ & $-0.08(9)$ \\
20 & $8.6(2.0)$ & $-0.10(9)$ & $8.5(1.8)$ & $-0.09(9)$ \\
30 & $9.6(2.4)$ & $-0.18(9)$ & $9.5(2.6)$ & $-0.10(8)$ \\
40 & $7.3(1.7)$ & $-0.10(5)$ & $9.8(3.8)$ & $-0.14(9)$ \\
50 & & & $8.3(1.1)$ & $-0.01(8)$ \\
60 & & $9.0(1.7)$ & $-0.10(9)$ \\
70 & & $7.2(1.2)$ & $0.01(9)$ \\
80 & & & $6.4(1.6)$ & $0.06(9)$ \\
\hline
\end{tabular}

TABLE II. Calculated surface tension and bending modulus for uniform bilayers. Figures in parenthesis give the estimated error in the final digit(s). For $\sigma$ the error was taken to be the standard error of the mean (taken over 25000 measurements).

\section{PARTICLES ADHERE TO BOUNDARIES IN PHASE SEPARATED BILAY- ERS}

Shown in Fig. 4 are representative end simulation snapshots for NE particles on phase separated bilayers. As may been they still have a tendency to cluster together with these clusters tending to adsorb onto the boundary between the two bilayer components. This is possibly driven by the decrease in the interfacial free energy. The competition between the effective attraction between the nanoparticles and between the nanoparticle and interface acts to reduce the size of clusters formed. For small numbers of nanoparticles this competition between interfacial adsorption and nanoparticle aggregation decreases the clustering of the nanoparticles, which is evident from the RDF (Fig. 5(a)), for which the first peak decreases substantially. For higher numbers of particles the clustering of nanoparticles at short distances is largely unchanged but there is significantly less structure in the RDFs at larger separations. This weaker interaction is reflected in the distribution of nanoparticle cluster sizes (Fig. 5(b)); for the uniform bilayer the nanoparticles form a few (one to three) larger clusters, whereas for the phase separated the nanoparticles form a larger number of 

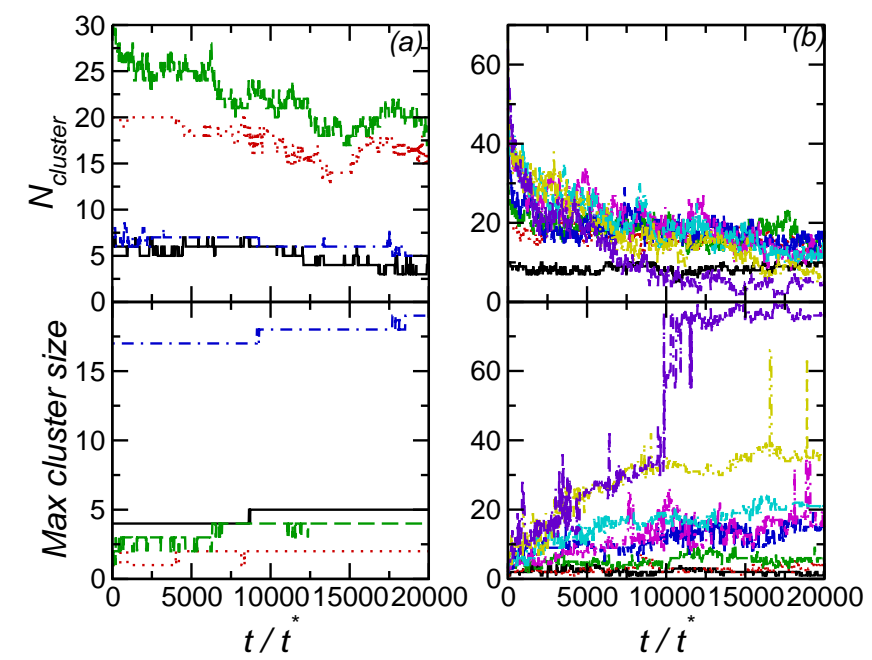

FIG. 3. (a) Time series of number of nanoparticle clusters (top) and largest cluster size (bottom) for NE nanoparticles. (b) Time series of number of nanoparticle clusters (top) and largest cluster size (bottom) for NI nanoparticles.

smaller clusters.

In contrast to the single component bilayer nanoparticles in the interior of a phase separated bilayer show little tendency to cluster in two component bilayers. Instead they adhere individually onto the boundary between the two components. The structures of the bilayers (shown in Fig. 6) resemble a two-dimensional analogue of a particle stabilised emulsion ${ }^{39}$. The adsorption of nanoparticles onto the boundary between the two bilayer components boundary between the two components creates closely packed nanoparticle layers. These layers inhibit domain coalescence increasing the stability of smaller domains. The tendency for particles to adhere individually is reflected in the RDFs (Fig. 7(a)). For small numbers of particles the first peak is significantly smaller than for the uniform bilayer and there is a complete lack of structure beyond this. For larger particle numbers some additional structure is present in the RDF at larger $r$, however, this is much weaker than in the single component bilayer. This suggests that the decrease in interfacial free energy caused by nanoparticle adsorption at the boundary between the bilayer components is greater than the weak attractive interaction that drives nanoparticle aggregation. For small numbers of particles the nanoparticle cluster size distribution is largely identical for both uniform and phase separated bilayers (Fig. 7(b)), which differs from the exterior case. As $N_{\text {par }}$ increases differences between the cluster size distributions for the uniform and phase separated bilay- 

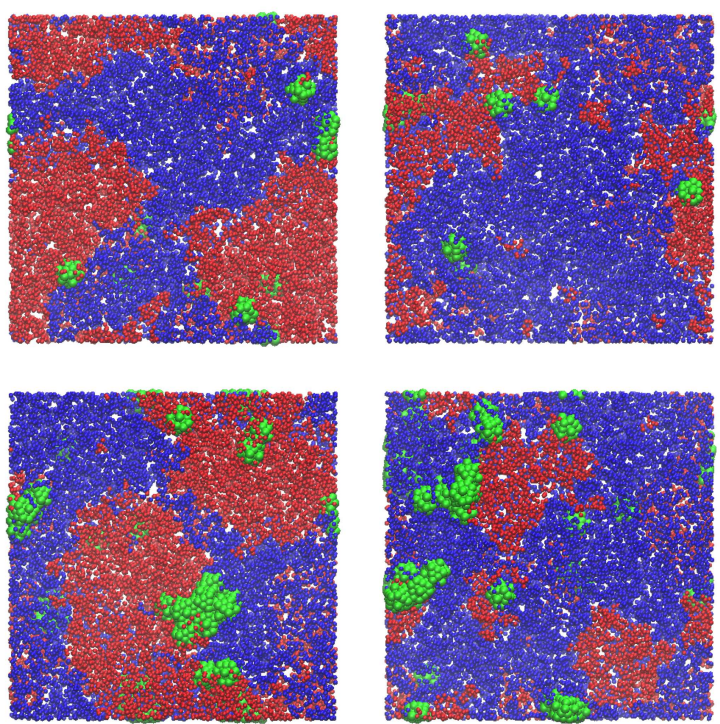

FIG. 4. Simulation snapshots showing representative configurations for NE nanoparticles on phase separated bilayers with (top to bottom) $N_{\text {par }}=10$ and $x_{b}=0.50, N_{p a r}=40$ and $x_{b}=0.50$, $N_{\text {par }}=10$ and $x_{b}=0.25$, and $N_{\text {par }}=40$ and $x_{b}=0.25$. Blue, red, and green spheres denote A-head, B-head, and NP beads respectively.

ers appear, with fewer large clusters forming for the phase separated systems, again caused by competition between cluster formation and adsorption at the boundary.

In order to rationalise the difference between the aggregation of nanoparticles in the two cases it is useful to compare the free energy of adsorption of a nanoparticle on the boundary to the interaction strength between nanoparticles. From simulation of completely phase separated bilayers the line tension between the two components may be estimated from the difference between the normal and transverse pressures (in the bilayer plane)

$$
\tau=\frac{1}{2 L_{x} L_{z}}\left(P_{y y}-P_{x x}\right)
$$

where the interface between the two-components is parallel to the $x$-axis. Using this the line tension between the two components is $\tau=0.13 k_{\mathrm{B}} \operatorname{Tr}_{c}^{-1}$ for $A_{H H^{\prime}}=90.0 k_{\mathrm{B}} \operatorname{Tr}_{c}^{-} 1$ and $\tau=3.92 k_{\mathrm{B}} \operatorname{Tr}_{c}^{-1}$ for $A_{T T^{\prime}}=85.0 k_{\mathrm{B}} T r_{c}^{-} 1$. Estimating the free energy of adsorption of a single nanoparticle as

$$
\Delta F_{a d s}=2 \tau R
$$



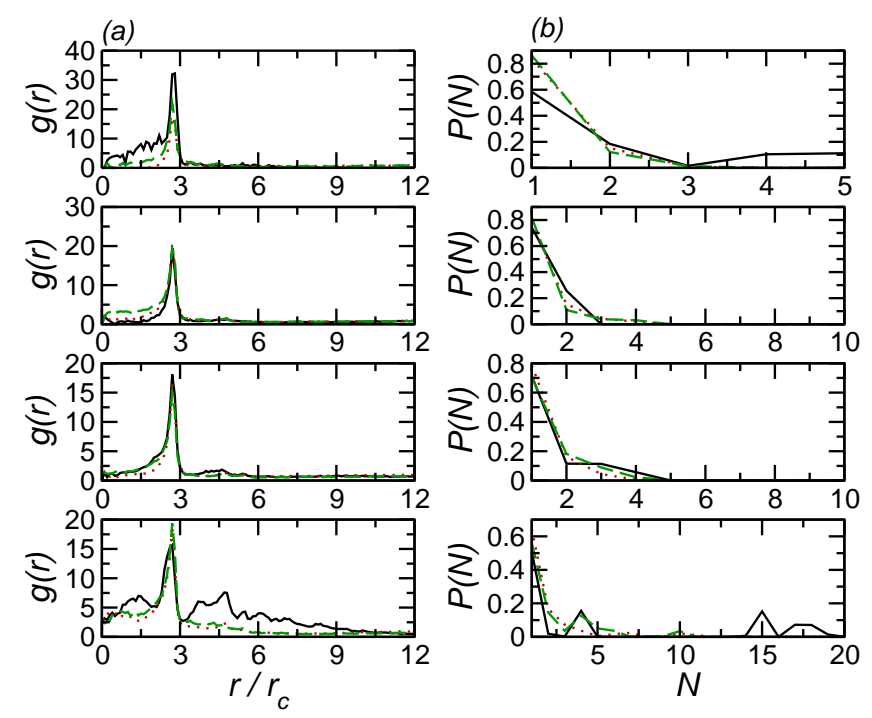

FIG. 5. (a) RDF for NE particles on mixed bilayers for (top to bottom) $N_{\text {par }}=10, N_{\text {par }}=20$, $N_{\text {par }}=30$, and $N_{\text {par }}=40$. In all cases solid line (black) denotes uniform bilayer, dotted line (red) denotes $x_{b}=0.50$, and dashed line (green) denotes $x_{b}=0.25$. (b) Nanoparticle cluster size distributions for NE particles on mixed bilayers for (top to bottom) $N_{\text {par }}=10, N_{\text {par }}=20$, $N_{\text {par }}=30$, and $N_{\text {par }}=40$. Symbols as in (a).

where $R=1.5 r_{c}$ is the nanoparticle radius. Using this gives $\Delta F_{a d s}=0.38 k_{\mathrm{B}} T$ and $11.80 k_{\mathrm{B}} T$ for unlike heads and tails respectively. The interaction strength between the nanoparticles may be estimated from the potential of mean force, which in the limit of low nanoparticle concentrations may be found from

$$
P M F(r)=-k_{\mathrm{B}} T \log g(r)
$$

The strength of the interaction between two nanoparticles can be estimated from the different between the PMF at first peak in the RDF and at infinite separation; for nanoparticles on the exterior of a uniform bilayer we then have $\triangle P M F=4.82 k_{\mathrm{B}} T$ while for nanoparticles in the bilayer interior we have $\triangle P M F=2.54 k_{\mathrm{B}} T$. For particles in the bilayer interior this interaction strength is significantly smaller than the adsorption free energy, which is consistent with the adsorption of nanoparticles individually onto the boundary. By contrast the interaction strength between nanoparticles on the bilayer exterior is larger than the adsorption free energy for individual nanoparticles so in this case the nanoparticles remain bound into clusters, which adsorb onto the boundary (the adsorption free energy of these 



FIG. 6. Simulation snapshots showing representative configurations for NI nanoparticles on phase separated bilayers with (top to bottom) $N_{\text {par }}=10, N_{\text {par }}=40$ and $N_{\text {par }}=80$. Snapshots for $x_{b}=0.50$ in the left hand column, snapshots for $x_{b}=0.25$ in right hand column. For clarity head beads are omitted (note white areas denote regions where head beads are in the bilayer interior and do not correspond to pores or voids in the bilayer). Yellow, purple, and black spheres denote A-tail, B-tail, and NP beads respectively.

clusters being larger than individual nanoparticles by virtue of their larger size).

\section{PARTICLES CAN NUCLEATE DOMAINS IN MIXED BILAYERS}

Whereas particles without an affinity for one of the bilayer components tend to adsorb onto the boundary between them, particles that preferentially interact with one of the com- 




FIG. 7. (a)RDF for NI particles on mixed bilayers for (top to bottom) $N_{\text {par }}=10, N_{\text {par }}=20$, $N_{\text {par }}=30, N_{\text {par }}=40$, and $N_{\text {par }}=80$. In all cases solid line (black) denotes uniform bilayer, dotted line (red) denotes $x_{b}=0.50$, and dashed line (green) denotes $x_{b}=0.25$. (b) Nanoparticle cluster size distributions for NE particles on mixed bilayers for (top to bottom) $N_{p a r}=10, N_{p a r}=20$, $N_{\text {par }}=30, N_{\text {par }}=40$, and $N_{\text {par }}=80$. Symbols as in (a).

ponents tend to remain in the bulk of their favoured phase. For bilayers composed of immiscible components non-neutral particles have little effect on the phase separation, with the particles remaining far from the interface. For miscible polymers, however, nanoparticles with an affinity for one phase nucleate domains of their favoured phase around them, as is shown in Fig. 8. Such behaviour is similar to that seen by Reynwar and Deserno ${ }^{27}$ for model proteins on a symmetric, near-critical mixed bilayer. Experimentally the ability of proteins to induce phase separation in mixed bilayers has been observed in a number of systems ${ }^{40,41}$.

This formation of small domains affects the clustering of nanoparticles. For $N_{\text {par }} \geq 20$ the peak in the RDF is larger for the two-component bilayers than in the uniform case (Fig. 9(a)). This suggests the presence of an attractive composition-mediated interaction, in agreement with previous simulations ${ }^{27}$. For intermediate numbers of particles this also 

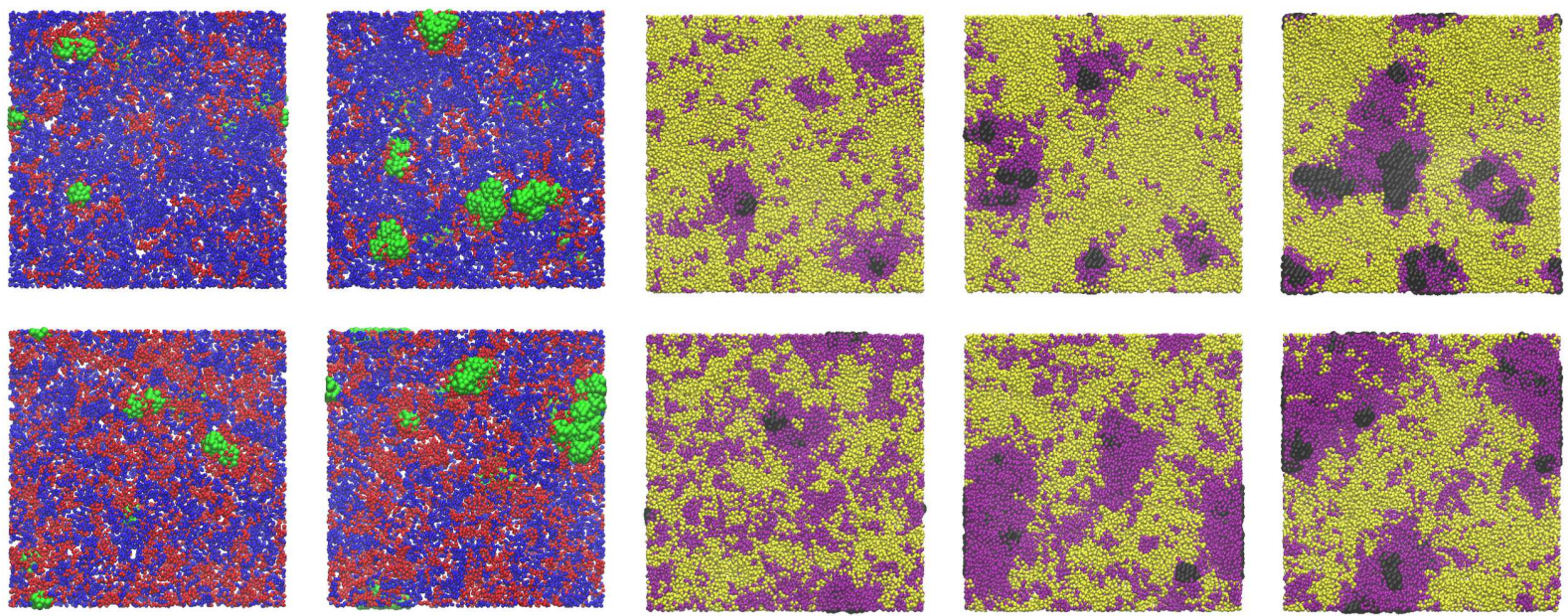

FIG. 8. Simulation snapshots showing representative configurations for (left to right) BE nanoparticles with $N_{\text {par }}=10$, BE nanoparticles with $N_{\text {par }}=40$, BI nanoparticles with $N_{\text {par }}=10$, BI nanoparticles with $N_{\text {par }}=40$, and BI nanoparticles with $N_{\text {par }}=80$. Top shows $x_{b}=0.25$, bottom shows $x_{b}=0.50$. Colours as in Figs. 4 and 6 .

leads to the formation of slightly larger nanoparticle clusters (Fig. 9(b)). Interestingly for the largest particle number studied the clusters formed in the two-component bilayer are smaller than in the uniform case. This suggests that the clustering may be size-limited possibly due to competition between the tendency for the effective attraction between the particles and B-heads leading and entropic gain in having the two components uniformly mixed.

For particles in the bilayer interior the change in the RDF with increasing particle number is quite different (Fig. 10(a)). For small particle numbers the peak in the RDF is significantly larger in the two-component case than for uniform bilayers, suggesting that the composition meditated interaction may be stronger in this case. This may be expected as the longer tails give rise to a stronger repulsion between unlike bilayer components (as is also evidenced by the smaller repulsion strength needed to induce demixing). In these cases some additional peaks in the RDF, absent in the uniform case, appear. As $N_{\text {par }}$ increases the difference between these decreases and for $N_{\text {par }}=80$ this is reversed. As $N_{\text {par }}$ increases the structure seen in the RDF at larger separations becomes less pronounced for the mixed bilayers. For all $N_{\text {par }}$ particles in the mixed bilayer form larger clusters than in the uniform case (Fig. 10(b)), 



FIG. 9. (a) RDF for BE nanoparticles with (top to bottom) $N_{\text {par }}=10, N_{\text {par }}=20, N_{\text {par }}=30$, and $N_{\text {par }}=40$. Solid line (black) denotes uniform bilayer, dotted line (red) denotes $x_{b}=0.50$, and dashed line (green) denotes $x_{b}=0.25$. (b) Nanoparticle cluster size distributions for BE nanoparticles with (top to bottom) $N_{\text {par }}=10, N_{\text {par }}=20, N_{\text {par }}=30$, and $N_{\text {par }}=40$. Symbols as in (a).

again suggestive of stronger composition mediated interactions.

\section{CONCLUSIONS}

Using dissipative particle dynamics simulations the aggregation of nanoparticles on one and two component model bilayers has been studied. For single component bilayers the aggregation of particles depends strongly on whether they reside in the bilayer exterior or interior. Particles on the bilayer exterior exhibit a strong attraction, which leads to the formation of tightly bound clusters, which remain bound over long timescales. Particles in the bilayer interior form less strongly bound, more dynamic clusters, suggesting that the inter particle interactions are weaker in this case. For small $N_{\text {par }}$ particles tend to exist as isolated particles, with only a few larger aggregates, while for the largest number of particles they form a single large cluster, with a few isolated particles, as would be expected for two-dimensional aggregation.

When the bilayer consists of two immiscible components the particles without an affin- 




FIG. 10. (a) RDF for BI nanoparticles with top to bottom) $N_{\text {par }}=10, N_{\text {par }}=20, N_{\text {par }}=30$, $N_{\text {par }}=40$, and $N_{\text {par }}=80$. Solid line (black) denotes uniform bilayer, dotted line (red) denotes $x_{b}=0.50$, and dashed line (green) denotes $x_{b}=0.25$. (b) Nanoparticle cluster size distributions for BI nanoparticles with top to bottom) $N_{\text {par }}=10, N_{\text {par }}=20, N_{\text {par }}=30, N_{\text {par }}=40$, and $N_{\text {par }}=80$. Symbols as in (a).

ity to either component tend to cluster at the boundary between these. Particles on the bilayer exterior remain bound in clusters with these clusters attaching to the boundary. The tendency of particles to cluster is weaker than on single component bilayers, possibly due to competition between particle clustering and adsorption onto the boundary. Due to their weaker interactions formation of clusters is suppressed for nanoparticles in the bilayer interior with these instead forming lines along the boundary, acting in a similar manner to nanoparticles at fluid interfaces in three dimensions ${ }^{39}$. This suggests that the desire to reduce the interfacial free energy (due to the line tension between the two bilayer components) is stronger than particle-particle interaction.

For bilayers composed of two miscible components the addition of nanoparticles with an affinity towards one component can lead to nucleation of small domains of their favoured 
phase around them, consistent with experimental simulation studies of proteins in mixed lipid bilayers. For particles in the bilayer exterior this leads to an increase in the attraction between the nanoparticles. This, however, is accompanied by a decrease in the ordering at longer range, with similar behaviour being seen for particles in the bilayer interior. While only one value of the repulsion between unlike polymers (i.e. $A_{H H^{\prime}}$ or $A_{T T^{\prime}}$ ) for each of the cases is presented here, preliminary simulations for unlike polymers without an excess repulsion (i.e. $A_{H H^{\prime}}=A_{H H}=A_{H^{\prime} H^{\prime}}$ and $A_{T T^{\prime}}=A_{T T}=A_{T^{\prime} T^{\prime}}$ ) have shown that in this case nanoparticles with an affinity for one component may also nucleate domains. How the sizes of these domains vary with the repulsion between the unlike polymers and how sensitive this is to the nanoparticle-polymer interaction will be investigated more fully in future work.

This work demonstrates that composition changes and ordering of nanoparticles on multicomponent bilayer membranes are coupled and that this may be used to control the aggregation of nanoparticles adsorbed on them. In particular the formation of linear and circular arrays of nanoparticles at the boundary between demixed polymer membranes may be used to form nanostructures for optical or electronic applications ${ }^{42}$. Particles with an affinity for one bilayer component cluster together due to composition driven interactions, which may be used to template the formation of polymer domains. Although this work has demonstrated this possibility future work will more fully explore how the nucleation of these domains is affected by chaining the affinity of the particles for the different components and the repulsion between the unlike components. This paper has focused on the aggregation of nanoparticles on bilayers it may be expected that nanoparticles may be used to control phase separation in mixed bilayers. While study of the interfacial length and other quantities suggest that this is possible the results are still inconclusive (not shown here) and more exhaustive simulations, using constant surface tension rather than surface area simulations, would be necessary to fully explore this. This would also allow for the investigation of the effect of bilayer flexibility on the phase behaviour and ordering of nanoparticles.

\section{ACKNOWLEDGEMENTS}

Some of these results were obtained using the EPSRC funded ARCHIE-WeSt High Performance Computer (EPSRC grant no. EP/K000586/1). Simulation snapshots were created 
using $\mathrm{VMD}^{43}$

* david.cheung@strath.ac.uk

1 D. E. Discher and A. Eisenberg, Science 297, 967 (2002).

2 J. R. Young, H. Andruleit, and I. Probert, Journal of Phycology 45, 213 (2009).

3 U. B. Sleytr, E. M. Egelseer, N. Ilk, D. Pum, and B. Schuster, FEB-Journals 274, 323 (2007).

4 X. Zhang, P. Tanner, A. Graff, C. G. Palivan, and W. Meier, Journal of Polymer Science Part A: Polymer Chemistry 50, 2293 (2012).

5 D. A. Christian, A. Tian, W. G. Ellenbroek, I. Levental, K. Rajagopal, P. a. Janmey, A. J. Liu, T. Baumgart, and D. E. Discher, Nature materials 8, 843 (2009).

6 D. A. Pantano, M. L. Klein, D. E. Discher, and P. B. Moore, Journal of Physical Chemistry B 115, 4689 (2011).

7 M. Turner, P. Sens, and N. Socci, Physical Review Letters 95, 168301 (2005).

8 S. Meinhardt, R. L. C. Vink, and F. Schmid, Proceedings of the National Academy of Sciences 110, $4476(2013)$.

9 M. Schulz, A. Olubummo, and W. H. Binder, Soft Matter 8, 4849 (2012).

10 R. Chen, D. J. G. Pearce, S. Fortuna, D. L. Cheung, and S. A. F. Bon, Journal of the American Chemical Society 133, 2151 (2011).

11 W. H. Binder, R. Sachsenhofer, D. Farnik, and D. Blaas, Physical chemistry chemical physics 9, 6435 (2007).

12 B. Wang, L. Zhang, S. C. Bae, and S. Granick, Proceedings of the National Academy of Science 105, 18171 (2008).

13 J. B. Hutchison, R. M. Weis, and A. D. Dinsmore, Langmuir 28, 5176 (2012).

14 S. U. Pickering, Journal of Chemical Society Transactions 91, 2001 (1907).

15 Y. Yan, G. K. Such, A. P. R. Johnston, J. P. Best, and F. Caruso, ACS Nano 6, 3663 (2012).

16 Y. Li, X. Chen, and N. Gu, Journal of Physical Chemistry. B 112, 16647 (2008).

17 H.-M. Ding, W.-d. Tian, and Y.-Q. Ma, ACS Nano 6, 1230 (2012).

18 T. H. F. Thake, J. R. Webb, A. Nash, J. Z. Rappoport, and R. Notman, Soft Matter 9, 10265 (2013).

19 J. P. Prates Ramalho, P. Gkeka, and L. Sarkisov, Langmuir 27, 3723 (2011). 
20 A. Alexeev, W. E. Uspal, and A. C. Balazs, ACS Nano 2, 1117 (2008).

21 T. Yue and X. Zhang, ACS Nano 6, 3196 (2012).

22 A. Šarić and A. Cacciuto, Soft Matter 9, 6677 (2013).

23 A. Šarić and A. Cacciuto, Physical Review Letters 108, 118101 (2012).

24 P. G. Dommersnes and J. Fournier, European Physical Journal B 12, 9 (1999).

25 R. Matthews and C. N. Likos, Physical Review Letters 109, 178302 (2012).

26 R. Matthews and C. N. Likos, Soft Matter 9, 5794 (2013).

27 B. J. Reynwar and M. Deserno, Biointerphases 3, FA117 (2008).

28 P. J. Hoogerbrugge, J. M. V. a. Koelman, and A. B. Rijswijk, Europhysics Letters (EPL) 19, 155 (1992).

29 R. D. Groot and P. B. Warren, Journal of Chemical Physics 107, 4423 (1997).

30 R. D. Groot and K. L. Rabone, Biophysical Journal 81, 725 (2001).

31 A. Maiti and S. McGrother, The Journal of chemical physics 120, 1594 (2004).

32 K. P. Travis, M. Bankhead, K. Good, and S. L. Owens, The Journal of chemical physics 127, 014109 (2007).

33 S. Plimpton, Journal of Computational Physics 117, 1 (1995).

34 J. N. Israelachvili, Intermolecular and surface forces (Academic Press, 2011), 3rd ed.

35 M. Goulian, R. Bruinsma, and P. Pincus, Europhysics Letters 22, 145 (1993).

36 J. Fournier and P. G. Dommersnes, Europhysics Letters 39, 681 (1997).

37 E. Lindahl and O. Edholm, Biophysical Journal 79, 426 (2000).

38 W. K. den Otter and W. J. Briels, The Journal of Chemical Physics 118, 4712 (2003).

39 B. P. Binks, Current Opinion in Colloid \& Interface Science 7, 21 (2002), ISSN 13590294.

40 A. T. Hammond, F. A. Heberle, T. Baumgart, D. Holowka, B. Baird, and G. W. Feigenson, Proceedings of the National Academy of Sciences of the United States of America 102, 6320 (2005).

41 T. Baumgart, S. T. Hess, and W. W. Webb, Nature 425, 821 (2003).

42 C. H. Lee, A. J. Crosby, R. C. Hayward, and T. Emrick, ACS applied materials \& interfaces 6, 4850 (2014), ISSN 1944-8252.

43 W. Humphrey, A. Dalke, and K. Schulten, Journal of Molecular Graphics 14, 33 (1996). 